S. Diefenbach, N. Henze \& M. Pielot (Hrsg.): Mensch und Computer 2015 Tagungsband, Stuttgart: Oldenbourg Wissenschaftsverlag, 2015, S. 443-446.

\title{
persona.fractalis - eine körpergesteuerte 3D-Installation
}

\author{
O. Köse, C. Mayer, F. Bünting, C. Geiger
}

Hochschule Düsseldorf, Mixed Reality und Visualisierung, geiger@hs-duesseldorf.de

\section{Zusammenfassung}

persona.fractalis ist ein audio-visuelle Medieninstallation, die es erlaubt in Echtzeit durch personalisierte 3D-Fraktale zu navigieren. 3D-Fraktale sind eine dreidimensionale Visualisierung mathematischer Funktionen auf der komplexen Zahleneben, die sich durch ihre Selbstähnlichkeit und unendliche Komplexität auszeichnen. Das System generiert auf Basis ausgewählter biometrischer Parameter des Nutzers in Echtzeit eine visuelle Darstellung mit hoher Auflösung (4K, 60 fps) bzw. in immersiver Darstellung (Oculus Rift). Parallel zur Visualisierung wird ambienter Sound auf Basis einer algorithmischen Komposition synthetisiert, was ebenfalls in Abhängigkeit der Nutzerdaten erfolgt. Das Mapping verschiedener biometrischer Sensorwerte erlaubt eine vielfältige Einflussnahme auf die Darstellung des Fraktals und ergibt eine individuelle Nutzungssituation. Durch Speichern der aktuellen Parameter lässt sich so eine personalisierte Medienerfahrung festhalten und später wieder abrufen.

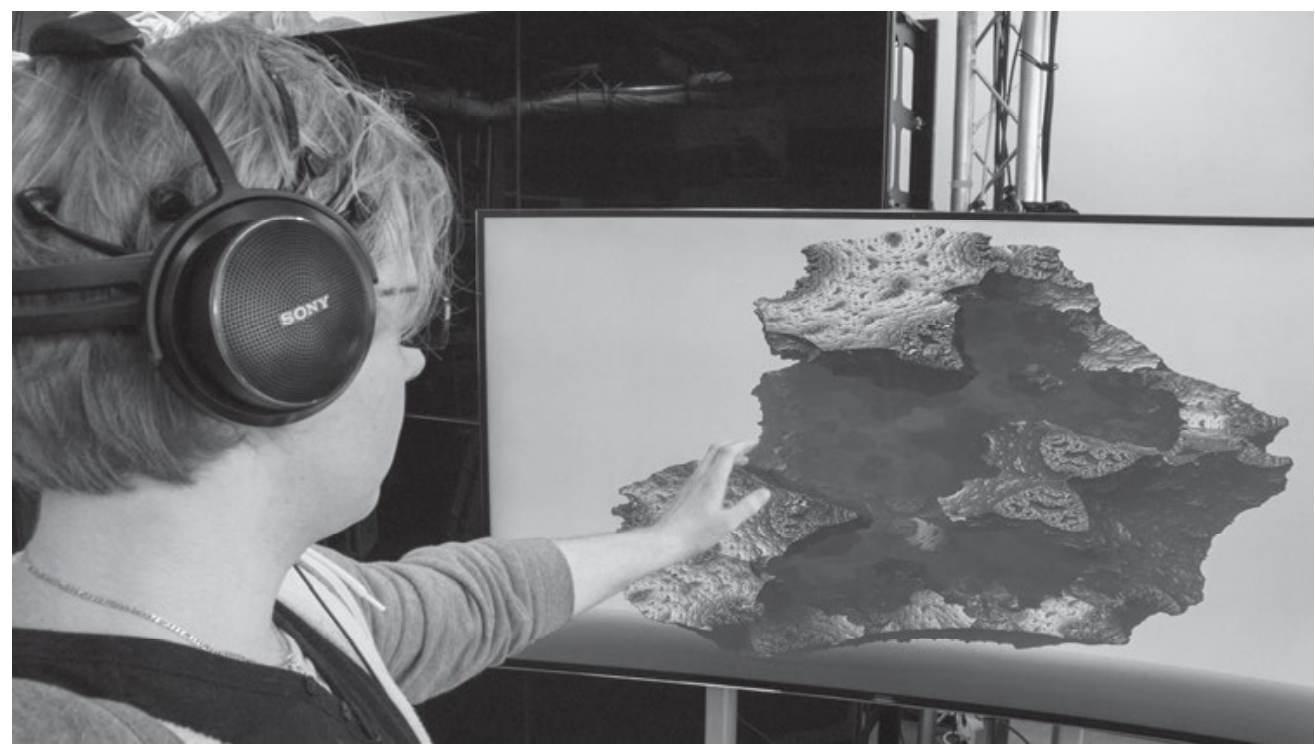

Abbildung 1: persona.fractalis auf einem 4K 65-Zoll 3D-Display mit Steuerung durch EEG-Daten und Gesten . 


\section{Einleitung und Konzept}

Natürliche Interaktion und die Nutzung alternativer Sensorik bzw. innovativer Eingabegeräte jenseits von Maus und Tastatur sind eine Kerneigenschaft moderner Medieninstallation, Computerspiele und experimenteller Mensch-Technik-Schnittstellen. Diese Technologien werden in diesem Projekt eingesetzt um die individuellen Körperdaten und -aktivitäten des Nutzers auf ein 3D-Fraktal abzubilden. Konkretes Ziel der Konzeption und Entwicklung von persona.fractalis war die Entwicklung einer interaktiven Medieninstallation, die auf Basis von Bewegungsdaten wie Position, Gesten bzw. Körperdaten wie Temperatur, Herzschlag und EEG die Darstellung eines 3D-Fraktals in Echtzeit ermöglicht und parallel dazu einen ambienten Soundteppich algorithmisch generiert. Dabei ermöglicht die Abbildung von Nutzerdaten auf Systemparameter eine für jeden Benutzer individuelle Visualisierung und Auralisierung.

\section{3D-Fraktalen in Echtzeit}

Klassische Fraktale wie die Mandelbrot- oder Juliamenge sind ursprünglich zweidimensional definiert (Mandelbrot 1991). Sie definieren eine Menge aus komplexen Zahlen, die bei der fortlaufenden Iteration einer Funktion bestimmte Kriterien erfüllen müssen, um der Menge anzugehören. Diese Zugehörigkeit kann auf einer zweidimensionalen Zahlenebene farblich dargestellt und somit ein 2D-Fraktal abgebildet werden. Die Mandelbrotmenge zeigt bespielweise eine geschlossene Fläche, dessen Rand jedoch unendlich komplex ist und somit bei einer Vergrößerung - des Randes - weitere Komplexität und Vielfalt zum Vorschein kommt. Die Iterationen bei der Erzeugung eines Fraktals führt zu einer weiteren Eigenschaft, nämlich der Selbstähnlichkeit: Teile des Fraktals ähneln dem Ganzen, wobei die Selbstähnlichkeit sehr stark, aber auch sehr schwächer ausgeprägt sein kann. In der Natur sind fraktale Formen sehr häufig anzutreffen. So besteht beispielsweise ein Baum aus einem Stamm von dem Äste abgehen, von denen wiederum kleinere Äste abgehen usw. Die Anzahl der Iterationen ist in der Natur begrenzt, kann jedoch in der Computergrafik beliebig oft durchgeführt werden.

Die Darstellung von 3D-Fraktalen kann mit einer Art Ray Tracing, dem Ray Marching, erfolgen. Dabei existieren Funktionen ${ }^{1}$, sogenannte "Distance Estimatoren", mit dessen Hilfe eine Approximation der komplexen dreidimensionalen Oberfläche und dadurch die Darstellung von 3D-Fraktalen ermöglicht werden. Das Ray Marching betrachtet jeden Pixel individuell und ermöglicht daher eine Parallelisierung des Algorithmus zur Berechnung der 3DFraktale. Aus diesem Grund kann die Berechnung der 3D-Fraktale mit Hilfe des Ray Marchings auf die GPU ausgelagert werden, wodurch die Interaktivität trotz hoher Auflösung gegeben ist (Köse 2015).

1 Auf der Seite http://www.fractalforums.com/ sind zahlreiche Funktionen für unterschiedliche 3D-Fraktale zu finden [02.Juli.2015]. 
Nach der Berechnung der fraktalen dreidimensionalen Geometrie können die Normalen und somit eine realistischere - und auch individuell bestimmbare - Illumination berechnet werden. Weil die Berechnungen und somit die Darstellung eines 3D-Fraktals auf mathematischen Funktionen aufbaut, können ausgewählte Parameter manipuliert werden und so die fraktale Form verändern. Eine geringfügige Veränderung eines Parameters kann schon große Folgen auf die Form und die Ästhetik des 3D-Fraktals haben. Wenn diese Parameter über die Zeit hinweg variiert werden, können visuell attraktive Verformungen der fraktalen Formen erzeugt werden. Abbildung 2 zeigt z. B. ein 3D-Sierpinski-Fraktal in verschiedenen Formen, die durch die Veränderung eines Fraktal-Parameters der Basisfunktion entstehen.


Abbildung 2: Verformung des 3D-Sierpinski-Fraktals, durch die Veränderung eines Parameters.

\section{Algorithmische Soundsynthese}

Um die visuelle Darstellung der 3D Fraktale akustisch zu unterstützen und ein emotionales audiovisuelles Erlebnis zu schaffen, erzeugt persona.fractalis eine Klanglandschaft in Echtzeit. Dabei werden verschiedene algorithmische Ansätze benutzt, die je nach Fraktaltyp ausgewählt werden. Allen Themen gemeinsam ist die Abhängigkeit von mathematischen Parametern der visuellen Fraktalgenerierung. Diese Parameter werden durch Bildeigenschaften (Helligkeit, Farbton) sowie biometrischen Daten des Nutzers ergänzt und speisen verschiedene Soundgeneratoren, die kurz skizziert werden.

Thema "lamina": Die eingespeisten Variablen werden dazu genutzt, um eine Klangtextur aus drei Schichten zu erzeugen, die sich in drei Frequenzbereiche aufteilen (Höhen, Mitten, Tiefen). Jede Schicht besteht dabei aus zwei verschiedenen zuvor erzeugten Klängen, die in Abhängigkeit der Fraktalparameter gemischt werden. Die gewonnenen Bildinformationen werden genutzt, um zusätzliche Effekte zu steuern, wie Hall, Filter und Delays. Bei abrupten Änderungen der Fraktalparameter wird zusätzlich ein Sendeffekt aktiv, der die Änderungen der Soundeigenschaften hervorhebt. Die verwendeten Soundsamples bewegen sich dabei von harmonischen, legato-artigen Flächen bis hin zu verrauschten und verzerrten Klängen.

Thema "granulum": Für das zweite Konzept wird ein Granularsynthesizer verwendet (Truax 1988). Dieser wird von einer harmonischen, streicherähnlichen Klangtextur gespeist. In der 
Ausgangslage des passenden Fraktals wird diese Klangtextur fast unbearbeitet abgespielt. Verändert man jedoch die Parameter des Fraktals, so steigt auch sukzessiv die Komplexität des Sounds, da nun das Ausgangsmaterial in immer kleinere Soundteile zerlegt und neu zusammengesetzt abgespielt wird. Zusätzliche Effekte wie im ersten Konzept vervollständigen abschließend die Klanglandschaft.

Thema "strepitus": Das dritte und letzte kurz vorgestellte Konzept besteht aus der Nutzung von verschiedenen Drone- und Noisegeneratoren. Diese sind in der Lage, rein synthetisch Klangteppiche zu generieren ohne Soundsamples zu verwenden. Die meist in einer Vielzahl vorhandenen Parameter wurden so mit den Fraktal- sowie Bildparametern verknüpft, dass sich bei der Ausgangslage eine harmonische und passende Soundstruktur bildet. Bei Änderungen am Fraktal zerfallen aber auch diese Strukturen und die Soundgeneratoren führen zu einem abstrakten und disharmonischen Ergebnis.

Bei allen Varianten werden Kopfhörer eingesetzt, um die akustische Immersion und die Wirkung der Klanglandschaften zu steigern.

\section{Realisierung und Installation}

Die vorgestellten Konzepte haben in erster Linie das Ziel, eine interessante und reaktive audiovisuelle Nutzererfahrung zu erzeugen. Das System wurde im Rahmen einer Bachelorarbeit mit Unity3D entwickelt [2] und ist vollständig funktionsfähig. Aktuell experimentieren wir mit der Abbildung verschiedener Sensordaten auf die visuellen und akustischen Parameter der Installation. Als Eingabegeräte werden aktuell für die Bewegung / Navigation eine Kinect2 bzw. ein Myo Gesture Control Armband verwendet. Das individuelle Nutzerprofil verwendet aktuell EEG-Informationen (über ein BCI-Device wie Emotiv Epoc) und verschiedene Biosignale wie Hautleitwert, Puls und EMG. Die Ausgabe erfolgt für alle sichtbar auf einem 65" Curved 4K-Display bzw. für einzelne Benutzer auf einer Oculus Rift DK2.

\section{Literatur}

Mandelbrot, B.B. (1991). Fraktale Geometrie der Natur. Birkhäuser Verlag.

Köse, O.S. (2015). Interaktive echtzeitfähige 3D-Fraktale, Bachelorarbeit, FH Düsseldorf.

Truax, B. (1988). Real-time granular synthesis with a digital signal processor. Computer Music Journal, 12(2), p14-26 